\title{
Bispectral index monitoring during cardiopulmonary resuscitation repeated twice within 8 days in the same patient: a case report
}

\author{
Michael T. Pawlik • Timo F. Seyfried • \\ Christian Riegger $\cdot$ Werner Klingler $\cdot$ Christoph Selig
}

Received: 5 April 2008 / Accepted: 12 May 2008 / Published online: 19 June 2008

(C) Springer-Verlag London Ltd 2008

\begin{abstract}
Research on cardiac resuscitation has led to various changes in the techniques and drug administration involved in modern advanced life support. Besides improving primary cardiac survival, interest is increasingly focused on a favourable neurological outcome. However, until now there has been no on-site equipment to support the clinical observations of the cardiopulmonary resuscitation (CPR) team. Bispectral index (BIS) monitoring has been used for avoiding awareness during anaesthesia for many years. We report a case of a 68 -year-old patient suffering twice from cardiac arrest due to thromboembolism within a few days. While the first cardiac resuscitation was survived without neurological consequences, the patient died after the second event. Both resuscitation events were monitored using the BIS. We discuss the course of BIS values and their possible contribution to the prediction of outcome.
\end{abstract}

Keywords Cardiac arrest · Bispectral index .

Cerebral monitoring $\cdot$ Resuscitation

The views expressed in this paper are those of the author(s) and not those of the editors, editorial board or publisher.

M. T. Pawlik $(\bowtie) \cdot$ T. F. Seyfried

Department of Anaesthesiology, University of Regensburg,

Franz-Josef-Strauss-Allee 11,

93042 Regensburg, Germany

e-mail: michael.pawlik@klinik.uni-regensburg.de

C. Riegger $\cdot$ W. Klingler $\cdot$ C. Selig

Department of Anaesthesiology, University Hospital Ulm,

Ulm, Germany

\section{Introduction}

Research on cardiopulmonary resuscitation (CPR) has been intensively carried out over recent years and led to improved rates of successful resuscitation. However, the topic of interest is not only the ultimate outcome of whether patients are discharged from the hospital, but also the neurological outcome. In this context, there is also growing interest in using neurological monitoring during or after CPR. A processed EEG analysis computing the bispectral index (BIS) is one method for monitoring the level of consciousness during anaesthesia. The BIS value is a single numeric value on a scale from 0 to 100 . While values of 85-100 indicate consciousness, sedation occurs at levels ranging from 65 to 85 and general anaesthesia is titrated to values between 40 and 60 . BIS values below 30 are associated with burst suppression and isoelectric EEG. The spontaneous EEG will be inhibited once cerebral perfusion pressure is reduced below a critical value as a result of the protective mechanism that reduces the metabolic demands of neuronal ischaemia. A review of the literature revealed only eight case reports of using BIS monitoring during CPR published in the last 6 years, but none described BIS monitoring in only one patient undergoing double cardiac resuscitation within a few days.

\section{Case report}

We report the case of a 69-year-old man suffering twice from cardiac arrest within 8 days, both monitored with BIS, which revealed different results with different outcomes. The first cardiac arrest occurred on the haemato- 
logical ward. On arrival of the CPR team, the patient was gasping for air, had enlarged pupils, and primary pulseless electrical activity was the first heart rhythm identified on electrocardiography. The patient's history revealed a B-cell leukaemia and autoimmune haemolysis. After immediate intubation and ventilation, the patient received a total of $4 \mathrm{mg}$ of epinephrine during CPR. Spontaneous circulation returned after $13 \mathrm{~min}$ and the patient was transferred to the intensive care unit (ICU), where he needed extensive catecholamine treatment for the next $6 \mathrm{~h}$.

BIS monitoring was started $11 \mathrm{~min}$ after the cardiac arrest and showed an initial BIS value of 87 and an average value of $78 \pm 5$, a suppression ratio of 0 and a sufficient SQI (signal quality index, derived from impedance measurement of electrodes), which is considered to be above 50 (Fig. 1). The sedation regimen consisted of $20 \mathrm{mg}$ etomidate in the first 15 min of CPR; additionally propofol (between 80 and $160 \mathrm{mg} / \mathrm{h})$ and fentanyl $(157 \mu \mathrm{g} / \mathrm{h})$ were administered after return of spontaneous circulation. In the following $2 \mathrm{~h}$ the patient suffered several times from brief cardiac arrest, which required short periods of CPR and $200 \mathrm{MEq}$ of sodium bicarbonate to stabilize metabolism. Eight hours after the first CPR the catecholamine dosage could be stopped, and the patient emerged from anaesthesia without showing neurological deficiencies. A Mini-Mental State Examination showed 27 of 30 points, and the Cerebral Performance Category score was 2 . To detect the cause of the cardiac arrest, computed tomography (CT) angiography scan of the body was performed and showed incomplete, bilateral pulmonary embolism of single seg-

\section{BIS monitoring of surviving event}

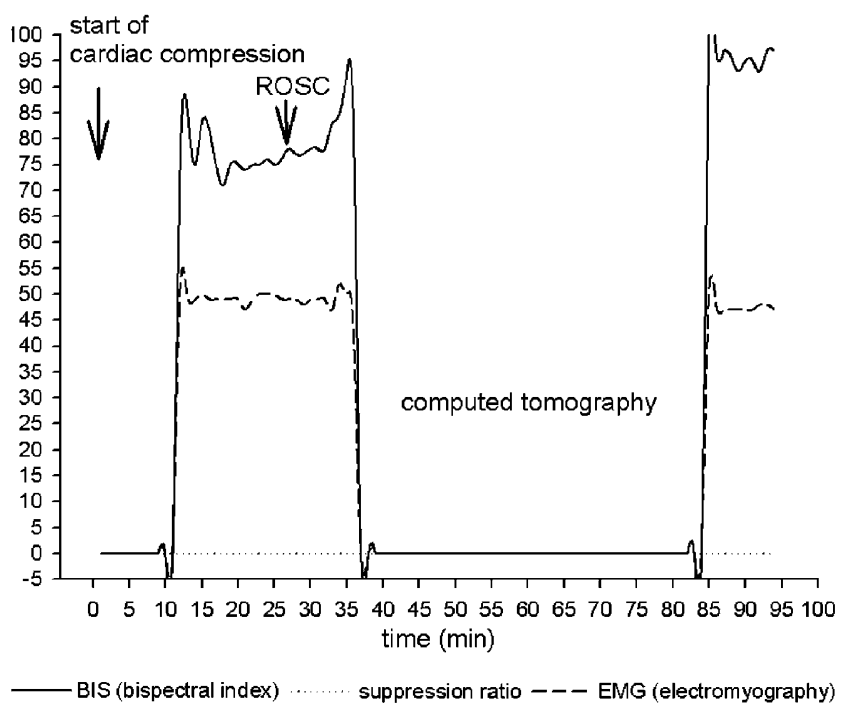

Fig. 1 BIS monitoring during the first cardiac arrest, when the patient survived without any neurological deficiency. BIS monitoring was interrupted for chest computed tomography. Note that the value of the suppression ratio was 0 during the entire external cardiac compression mental arteries as the probable cause of the cardiac arrest, while radiological examination disclosed no pathological findings in the brain. The patient was discharged from the ICU on day 4 after admission under stable cardiopulmonary conditions, but suffering from acute renal failure.

Eight days after discharge, the patient collapsed again and suffered from respiratory insufficiency with dyspnoea, cyanosis and an oxygen saturation of $70 \%$. The patient was transferred to the ICU with a suspected diagnosis of pulmonary embolism. It was possible to stabilize the patient under biphasic positive airway pressure (BIPAP) ventilation. No CT scan for the diagnosis of pulmonary embolism followed due to the lack of therapeutic consequences. After the first stabilization with non-invasive artificial ventilation the patient's respiration and circulation became increasingly insufficient, requiring intubation and catecholamine treatment. Sedatives and analgesics consisted of midazolam $(10 \mathrm{mg} / \mathrm{h})$ and fentanyl $(157 \mu \mathrm{g} / \mathrm{h})$. Later on, the patient again needed CPR after diagnosis of pulseless electrical activity on the ECG, probably caused by a second thromboembolic event. It was possible to maintain spontaneous circulation for a short period of time. BIS monitoring started $18 \mathrm{~min}$ after advanced cardiac life support. The initial BIS value was 72 and decreased to 13 during CPR with an average value of $46 \pm 22$ (Fig. 2). The suppression ratio increased from 11 to 68 and EMG decreased from 39 to 28. Despite intensive treatment with high catecholamine

\section{BIS monitoring of fatal event}

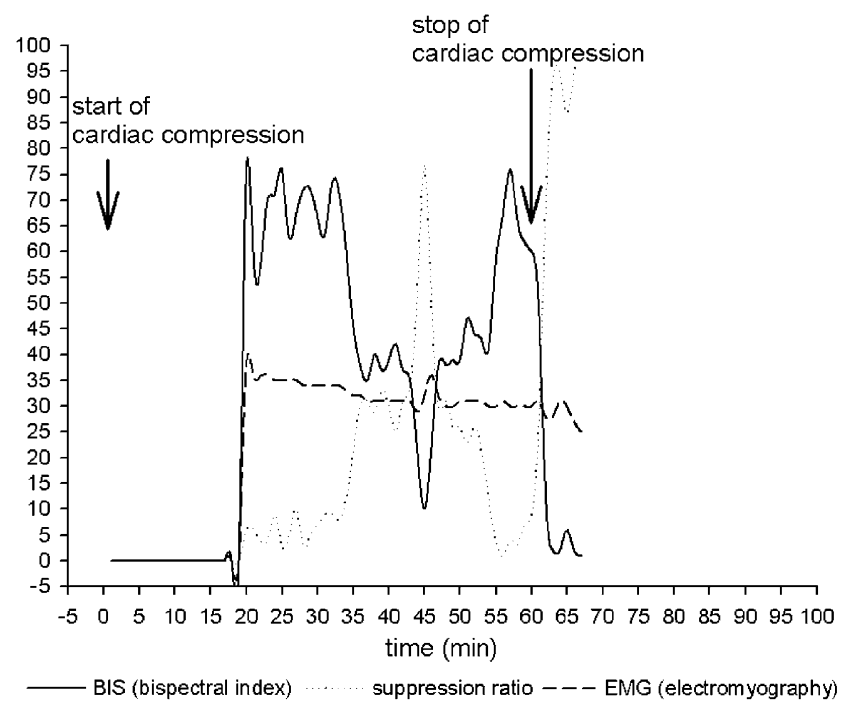

Fig. 2 The second cardiac arrest was followed by an unsuccessful cardiac resuscitation. BIS values initially reached high levels and decreased after $15 \mathrm{~min}$ of cardiac decompression. The burst suppression pattern occurred immediately at the beginning of resuscitation and suppression continuously rose until the end of cardiac compression. The last increase of BIS possibly represents improved cerebral activity following a rise of mean arterial pressure caused by a final bolus of $5 \mathrm{mg}$ epinephrine prior to stopping CPR 
doses, it was not possible to combat the haemodynamic insufficiency and thus intensive care physicians decided to stop CPR.

\section{Discussion}

BIS monitoring has shown its value in operating rooms around the world in preventing awareness during surgery and in the control of anaesthesia [1, 2]. There are a few reports of using BIS as a monitor in cardiac arrest and there is considerable interest in whether it could be useful to predict the outcome of CPR [3].

We report a case of a 69 -year-old patient with the unique situation of having had two cardiac arrests within 8 days, receiving BIS monitoring twice during the $\mathrm{CPR}$ and showing two different courses of BIS values, resulting in two completely different outcomes.

During the first event the patient had immediately high BIS values under CPR and a good neurological outcome. The CPR team considered stopping resuscitation due to the length of the resuscitation and the patient's palliative status, but the patient showed return of spontaneous circulation. Assuming that high BIS values result from good cerebral perfusion, the conclusion would be that high BIS values might be the result of a sufficient mechanical resuscitation. Additionally, BIS monitoring of the suppression ratio can also detect suppression patterns indicating cerebral ischaemia. During the first course of CPR the electrical activity of the patient's brain did not show any suppression ratio, when BIS monitoring started. This fact highlights the existing activity of basic and functional metabolism of the brain. In the second course of CPR 8 days later, the patient had low BIS values under CPR, which further decreased. The suppression ratio continuously increased during the unsuccessful second CPR.

The most interesting point seems to be that BIS monitoring may be a good parameter of sufficient cardiac output under cardiac massage in cases of pulmonary thromboembolism. During the first event fragmentation of the pulmonary thrombus apparently occurred resulting in good cerebral perfusion and BIS values. At the second event cardiac massage failed to result in sufficient cerebral perfusion. Previous reports have suggested BIS values above 40 as favourable for the patient's outcome [4]. Additionally, increasing BIS values in the early stage of both during and after resuscitation are associated with good survival and sufficient cerebral performance [5, 6].

Unlike former case reports, the bispectral index, suppression ratio and EMG values are presented for discussion in the context of a double CPR event in the same patient.

There are several concerns in the overestimation of pure BIS values, which could limit the prognostic value of BIS monitoring. Various manipulations to the head and body during CPR can lead to high EMG values, which can cause an artificial increase of the BIS and may diminish the assessment of BIS values. Furthermore, there is no fixed relationship between SQI, EMG and BIS changes. EMG levels between 40 and 60 may influence the BIS values and cause false high BIS values [7]. Additional difficulties in interpreting the BIS values may be caused by hypothermia and shivering.

A low signal quality and narcotics can also influence the BIS value, especially propofol, anaesthesia gas and other anaesthetics [8-10]. The BIS values of our patient increased despite sedation with etomidate and fentanyl during the first CPR and decreased during the second CPR under an even lower amount of sedatives. Additionally, etomidate in particular is known to cause muscle fasciculations, which can produce an EMG artifact [11]. However, the administered drugs did not have a strong influence on the BIS and EMG values in the dose in which they were applied in both CPRs, and the EMG remained at the same level despite the application of a small dose of etomidate. One limitation of interpreting BIS values may be a low EEG quality, but the signal quality was sufficient and high BIS values resulted in survival and a good cerebral outcome after the first CPR. Initially lower and further decreasing BIS values were associated with the death of the patient at the second CPR (Fig. 2).

The patient showed BIS values around 77, which resulted in a good outcome (Fig. 1), while values between 55 and 10 during CPR resulted in an unfavourable outcome (Fig. 2). Other authors described BIS values above 40 as acceptable during CPR $[3,12]$. Interestingly, the pattern of the suppression ratio showed a relatively high value at the beginning of monitoring, which continuously increased until the end of resuscitation during the second cardiac arrest. There may be a possible reciprocal correlation between the high suppression ratio values and the success of CPR. Presumably, the suppression ratio may give more information in combination with the BIS value than the latter value alone. Since the suppression ratio value cannot be influenced by the EMG, it may be a more reliable parameter than the BIS value, which is calculated from many subparameters including the EMG. However, increasing BIS values after stabilization of the circulation correlated with favourable outcome in this particular case, while decreasing values ended with death. Besides cerebral monitoring during anaesthesia BIS may monitor the success and quality of external cardiac compression especially in patients suffering from thromboembolism in the pulmonary circulation.

Funding There was no financial support received or author involvement with organizations with financial interests in the subject matter. 


\section{References}

1. Myles PS, Leslie K, McNeil J, Forbes A, Chan MT (2004) Bispectral index monitoring to prevent awareness during anaesthesia: the B-Aware randomised controlled trial. Lancet 363:1757-1763

2. Glass P (2002) Using the bispectral index for anaesthesia. Hosp Med 63:310

3. Fatovich DM, Jacobs IG, Celenza A, Paech MJ (2006) An observational study of bispectral index monitoring for out of hospital cardiac arrest. Resuscitation 69:207-212

4. Fabregas N, Gambus PL, Valero R, Carrero EJ, Salvador L, Zavala E, Ferrer E (2004) Can bispectral index monitoring predict recovery of consciousness in patients with severe brain injury? Anesthesiology 101:43-51

5. Shibata S, Imota T, Shigeomi S, Sato W, Enzan K (2005) Use of the bispectral index during the early postresuscitative phase after out-of-hospital cardiac arrest. J Anesth 19:243-246

6. Szekely B, Saint-Marc T, Degremont AC, Castelain MH, Fischler M (2002) Value of bispectral index monitoring during cardiopulmonary resuscitation. Br J Anaesth 88:443-444

7. Bruhn J, Bouillon TW, Shafer SL (2000) Electromyographic activity falsely elevates the bispectral index. Anesthesiology 92:1485-1487
8. van Twest RM (2006) Bispectral index guided timing of intubation without neuromuscular blockade during sevoflurane induction of anaesthesia in adults. Anaesth Intensive Care 34:606-612

9. Consales G, Chelazzi C, Rinaldi S, De Gaudio AR (2006) Bispectral Index compared to Ramsay score for sedation monitoring in intensive care units. Minerva Anestesiol 72:329-336

10. Fodale V, Pratico C, Santamaria LB (2004) Coadministration of propofol and midazolam decreases bispectral index value as a result of synergic muscle relaxant action on the motor system. Anesthesiology 101:799; author reply 800-801

11. Lallemand MA, Lentschener C, Mazoit JX, Bonnichon P, Manceau I, Ozier Y (2003) Bispectral index changes following etomidate induction of general anaesthesia and orotracheal intubation. Br J Anaesth 91:341-346

12. Chakravarthy M, Patil T, Jayaprakash K, Shivananda N, Jawali V (2003) Bispectral index is an indicator of adequate cerebral perfusion during cardiopulmonary resuscitation. J Cardiothorac Vasc Anesth 17:506-508

Dr. Pawlik is an instructor and attending physician in anaesthesiology at the University Medical Center in Regensburg, Germany. 